\title{
Die «Deutsche Periode» (etwa 1850-1880) in der Geschichte der Schweizer Psychiatrie und die moderne Sozialpsychiatrie*
}

Aus dem medizinhistorischen Institut der Universität Zürich

(Direktor: Prof.Dr.med.E.H.ACKeRKNECHT)

Von Hans H. Walser

Die Schweizer Psychiatrie hat um 1900 ihre charakteristische Form gefunden ${ }^{1}$. Ihre Begründer waren August Forel (1848-1931) und sein Schüler und Nachfolger Eugen Bleuler (1857-1939). Durch Adolf Meyer (1866-1931) erfuhr dieses hauptsächlich von Zürich ausgehende Gedankengut eine weltweite Verbreitung 2,3. Natürlich hat die «Zürcher Schule» historische Voraussetzungen, die zwar wenig beachtet wurden, aber sehr interessant sind. Nimmt man 1879, das Jahr des Amtsantrittes von August Forel an der Zürcher psychiatrischen Universitätsklinik, als Geburtsjahr der Schweizer Psychiatrie an, so findet man in den vorangehenden drei Jahrzehnten bereits eine recht fest etablierte praktische Psychiatrie und vor allem eine Fülle von fortschrittlichen Ideen vor. Diese haben mit den modernen sozialpsychiatrischen Bestrebungen oft eine erstaunliche Ähnlichkeit. Sie sollen deshalb unter dem doppelten Aspekt: der Vorbereitung der Schweizer Psychiatrie eigener Prägung einerseits und der modernen Sozialpsychiatrie andererseits, hier kurz dargestellt werden.

\section{Die Institutionen}

Die Entstehung einer Psychiatrie als medizinisches Spezialfach im letzten Jahrhundert erweist sich besonders stark an die meist staatlichen Hospitäler und Kliniken gebunden. Der Anstoß zur entscheidenden Verbesserung des Loses der Geisteskranken und zur wissenschaftlichen Psychiatrie kam

* Herrn Prof. Dr.med. Heinrich Buess zu seinem sechzigsten Geburtstage gewidmet.

1 Als charakteristisch dürfen etwa gelten: die biologische Grundhaltung, die gleichmäßige Anerkennung somatischer und rein psychischer Gesichtspunkte, die Betonung des Wertes der Psychotherapie, der Psychohygiene und der Sexologie, die frühe Ablehnung der Auswüchse der psychiatrischen Erblehre, die Therapiefreundlichkeit.

2 Manfred Bleuler, Early Swiss Sources of Adolf Meyer's Concepts, Amer. J. Psychiatry 119 (1962) Nr. 3.

3 HaNs Walser, Die wissenschaftlichen Anfänge von Adolf Meyer (1866-1950) und die Entstehung der «Zürcher psychiatrischen Schule», Gesnerus 23 (1966) 202-210. 
denn auch von einem Hospitaldirektor und aus Paris, wo die Spitalmedizin besonders stark ausgeprägt war ${ }^{4}$. Philippe Pinels «psychiatrische Reform» hatte denn auch zunächst keine großen Auswirkungen auf unser Land: In einem Bund von weitgehend autonomen Kleinststaaten waren die Voraussetzungen - Allgemeinhospitäler von einiger Größe - nur an wenigen Orten gegeben. Viele Landkantone taten bis zur Jahrhundertmitte wenig oder nichts für ihre Geisteskranken, während die größeren Stadtkantone in ihren Allgemeinhospitälern seit je auch Psychotische aufgenommen hatten ${ }^{5}$. Für diese wurden sogar gelegentlich spezielle, kleine Häuser mit «Tobzellen» errichtet, so etwa in Bern schon 1749, in Zürich 1817, in Basel noch 1838 6, 7, 8. Man könnte diese Zeit die «Periode der kleinen Irrenhäuser » nennen, sie wäre dadurch gekennzeichnet, daß die Geisteskranken in kleinen Spezialinstitutionen im Rahmen eines Allgemeinhospitales untergebracht waren. Um die Jahrhundertmitte wurde sie von der «Periode der Irrenanstalten» abgelöst. Damals entstanden neue und moderne Irrenanstalten, die getrennt von den Spitälern für körperlich Kranke errichtet wurden. Von St. Pirminsberg/St. Gallen und Préfargier/Neuchâtel (1847 bzw. 1848) über Waldau/Bern (1855) und Rheinau/Zürich (1867) bis zu Bel-Air/ Genève im Jahre $1900^{\circ}$. wurden in unserem Lande mit vorbildlicher Großzügigkeit rund zwanzig psychiatrische Kliniken erstellt, die die Träger des therapeutischen und wissenschaftlichen Fortschrittes wurden und bis heute Bestand haben. Wichtig wurde besonders die Zürcher Klinik Burghölzli (1870), weil hier die prominentesten Schweizer Psychiater, August Forel

4 E. H. Ackerknecht, Medicine at the Paris Hospital 1794-1848, The John Hopkins Press, Baltimore 1967.

5 Dieses Urteil ist nicht zu hart (siehe z. B.: J.M. HungerbüHLER, Über das öffentliche Irrenwesen in der Schweiz, St. Gallen/Bern 1846), wird aber der Vielfalt der Verhältnisse nicht ganz gerecht. Insbesondere hatte das von Paris beeinflußte Genf eine gewisse Sonderstellung (siehe z.B. E.R.Müllener, Pierre-Charles-Alexandre Louis' Genfer Schüler und die "méthode numérique», Gesnerus 24 (1967) 46-74.

6 W.Morgenthaler, Bernisches Irrenwesen, Bern 1915.

7 M.Hofmann, Die Irrenfürsorge im alten Spital und Irrenhaus Zürichs, Diss. med. Zürich 1922.

8 Madeleine Ziegler, Die Entwicklung des Irrenwesens in Basel (etc.), Diss. med. Basel 1933.

9 Das späte Datum für Genf erklärt sich wie dasjenige von Basel (Friedmatt eröffnet 1886) dadurch, daß diese Stadtkantone am Ende der «Periode der kleinen Irrenhäuser» in den dreißiger Jahren recht gut für die Geisteskranken gesorgt hatten, so daß sie den «neuen Anstaltstypus» erst später verwirklichen mußten. 
und Eugen Bleuler, wirkten und weil der junge Adolf Meyer hier seine grundlegende Ausbildung erfuhr ${ }^{10,11}$. «Zürcher Schule» und «Schweizer Schule» dürfen deshalb für jene Zeit, und für eine kurze Übersicht wie die vorliegende, synonym gebraucht werden.

\section{Die führenden Psychiater in der Vorbereitungsperiode}

Wer die Kulturgeschichte der Schweiz im 19.Jahrhundert kennt, wird nicht überrascht sein, daß die Voraussetzungen zur Schweizer Psychiatrie von deutschen, in unserem Lande wirkenden Nervenärzten geschaffen wurden $^{12}$. Für diese Vorbereitungszeit soll deshalb die Bezeichnung «Deutsche Periode» vorgeschlagen werden, besonders auch, weil der deutsche Einfluß gerade auf dem Gebiet der Psychiatrie besonders groß war. Diese Periode würde vom Beginn der großen Bauperiode psychiatrischer Kliniken in den 1840 er Jahren bis zu August Forel reichen. Glück war für unser Land einmal mehr dabei, denn gerade damals war die deutsche Psychiatrie - wie die deutsche Medizin überhaupt - in raschem Aufstieg begriffen. Die Schweiz, namentlich die jungen Universitäten Bern und Zürich, übten auf begabte junge deutsche Ärzte eine große Anziehungskraft aus, die reaktionäre Politik der deutschen Staaten ließ fortschrittlichen Männern den Aufenthalt bei uns ohnehin oft geraten erscheinen. Wenigstens die wichtigsten der Psychiater sollen hier genannt werden ${ }^{13}$ : Heinrich Ellinger wurde 1847 nach St.Pirminsberg berufen, 1856 gründete er eine Privatklinik, aus der die heutige psychiatrische Klinik Wil/St. Gallen hervorging. Ludwig BirsWANGer (1820-1880) leitete zuerst die thurgauische Anstalt Münsterlingen, dann seine Privatklinik Bellevue in Kreuzlingen, er ist der Stammvater einer eigentlichen Psychiaterdynastie. Heinrich Cramer (1831-1893) wurde 1861 Direktor von Rosegg/Solothurn, er wurde besonders bekannt als Experte für Anstaltsbauten und als Vorkämpfer des No-restraint, 1877 wurde er Professor für Psychiatrie in Marburg. August Zins (1825-1897) kam 1847 als politischer Flüchtling in die Schweiz, arbeitete 1854-1856 an

10 M.Bleulen (Herausgeber), Kantonale Psychiatrische Universitätsklinik Burghölzli Zürich 1870-1970, Zürich 1970.

11 M. Bleuler, Geschichte des Burghölzlis und der psychiatrischen Universitätsklinik, in Zürcher Spitalgeschichte, herausgegeben vom Regierungsrat des Kantons Zürich, 2.Band, Zürich 1951.

12 H. Ellenberger, La psychiatrie suisse, Aurillac (1951-1953).

13 Das Folgende zum Teil nach Тн. КікснноғF, Deutsche Irrenärzte, 2 Bände, Berlin 1924, zum Teil nach noch unpublizierten eigenen Studien. 
der alten Zürcher Irrenanstalt und war 1864-1872 Direktor von St. Pirminsberg. Auch er kehrte nach Deutschland zurück, wo er eine angesehene Stellung einnahm. Ludwig WiLle (1834-1912) war von 1864 an nacheinander Direktor der Anstalten Münsterlingen, Rheinau und St. Urban, 1875 wurde er Professor für Psychiatrie in Basel. Alle diese Männer aber wurden von Wilhelm Griesinger (1817-1868) weit in den Schatten gestellt. Er war 1860-1864 Professor für innere Medizin in Zürich, betätigte sich aber auch intensiv auf psychiatrischem Gebiet ${ }^{10,14}$. Schließlich waren BERNHARD von Gudden (1824-1886) und Eduard Hitzig (1838-1907) als Professoren für Psychiatrie in Zürich tätig (1868-1872, bzw. 1875-1879), beide sind als Forscher auf den Gebieten der Hirnanatomie bzw. der Hirnphysiologie hochbedeutend, haben aber für die praktische Psychiatrie, auf die es hier ankommt, bei uns weniger geleistet. Gudden hat den jungen August Forel für Psychiatrie zu begeistern vermocht, er und Theodor Meynert, Forels weiterer Lehrer, haben deshalb der Schweizer Psychiatrie gleichsam Pate gestanden. Recht interessant ist, daß schon der Assistent Forel Gudden gegenüber auf zwei Gebieten nachdrücklich seinen eigenen Standpunkt vertrat ${ }^{14}$ a : Auf dem Gebiet der Tierpsychologie (bzw. der biologisch begründeten Psychiatrie) und auf dem Gebiet des Somnambulismus (bzw. der Hypnose oder einer psychodynamischen Auffassung). - Natürlich ist es nicht so, als hätten Schweizer Psychiater in dieser Zeit überhaupt gefehlt, sie traten aber, wie etwa die Verhandlungen des 1864 gegründeten Vereins Schweizer Irrenärzte zeigen, an Bedeutung hinter ihren deutschen Kollegen zurück. Es sollen hier immerhin die Namen A.Tribolet, F. Brenner, R. Schärer, R.F. Fetscherin, E. Schaufelbühl und H. Henne genannt werden, die der zwischen 1794 und 1833 geborenen Generation angehören ${ }^{14}{ }^{\text {b }}$.

\section{Probleme beim Aufbau der praktischen Psychiatrie}

a) Die Trennung der psychiatrischen Anstalten von den Allgemeinhospitälern und die Schaffung eines psychiatrischen Anstaltstypus. Die alten Allgemeinhospitäler für körperlich und psychisch Kranke, für Gebrechliche und Greise konnten vom frühen 19. Jahrhundert an den Anforderungen einer neuen, naturwissenschaftlich orientierten Medizin nicht mehr genügen.

14 W.LöfFLER, Die medizinische Klinik Zürich 1833-1950, in Zürcher Spitalgeschichte, 2. Band, Zürich 1951.

${ }^{14 a}$ A. Forel Rückblick auf mein Leben, 2. Auflage, Zürich 1947.

14b Über FrIEDRICH BRENNER erscheint demnächst eine Basler Dissertation von G. Schüler (unter Leitung von H. Koelbing). 
Für die nun entstehenden Spezialgebiete wurden nach und nach besondere Kliniken errichtet, so z.B. in Zürich 1842 zunächst für Chirurgie, innere Medizin und Anatomie das neue Kantonsspital. Auch die Psychiatrie konnte sich im alten Rahmen nicht richtig entwickeln, wofür das noch 1838 im Basler Spitalareal eröffnete Irrenhaus zum warnenden Beispiel dienen kann ${ }^{15}$. Spezialkliniken für Geisteskranke bedeuteten für die damaligen Verhältnisse entschieden einen Fortschritt. Es wurde dafür ein neuer Anstaltstypus teils aus dem Ausland übernommen, teils neu gestaltet. Er umfaßte, unter Trennung der Geschlechter ${ }^{16}$, differenzierte, den Krankheitsformen angemessene Abteilungen mit Spazierhöfen, Möglichkeiten für die Arbeitstherapie, guten hygienischen Einrichtungen (Typhusfälle waren häufig!) sowie mit einer Zellenabteilung für Unruhige, denn chemische Beruhigungsmittel gab es noch kaum, und mechanischer Zwang war bei den fortschrittlichen Psychiatern verpönt. Diese Anstalten wurden getrennt von den Spitälern für Körperkranke errichtet. Das war ein entscheidender Schritt, denn das alte Allgemeinhospital bot bei all seinen Mängeln doch Fürsorge und Behandlung für sämtliche Leiden des Menschen - und für dessen soziale Bedürfnisse jedenfalls mehr, als die recht kahl und kalt wirkenden neuen Häuser ${ }^{17}$. Die Wiedervereinigung von Körpermedizin und Psychiatrie als Institutionen ist auch heute wieder aktuell ${ }^{18}$, ebenso die innere Gestaltung des Anstaltsmilieus in sozialpsychologischer Hinsicht. Entgegenzutreten ist jedenfalls der heute oft kritiklos wiederholten Behauptung, man habe mit den neuen psychiatrischen Anstalten lediglich den Geisteskranken von der Gesellschaft isolieren wollen, die Dinge liegen keineswegs so einfach.

b) Die Anstaltsleitung. In der neuen Anstalt sollte ein Arzt an der Spitze der gesamten ärztlichen und verwalterischen Aufgaben stehen, das war eine allgemeine Forderung der Ärzte. August Zinn hat sie unter Hinweis auf die Mißstände im alten Zürcher Irrenhaus besonders deutlich begrün-

15 A.Burckhardt, Geschichte der medizinischen Fakultät zu Basel 1460-1900, Basel 1917 (S. 295, Anm 5: C.G. Jung I. bezeichnete das alte Irrenhaus als «ursprünglich verpfuscht»).

16 Moderne Sozialpsychiater bezeichnen demgegenüber die Einteilung einer Klinik nach Geschlechtern als das Ungeeignetste, was man tun könne (A. UchtenHAGEN).

17 H. WALSER, Everyday Life in a European Hospital (etc.) J. Hist. Med. Allied Sci. 25 (1970) 22-30.

18 E. H. Ackerknecht und K. Akert, Wechselnde Formen der Unterbringung von Geisteskranken, Schweiz. med. Wschr. 94 (1964) 1541-1546. 
$\operatorname{det}^{19}$ : Bisher war nämlich der Verwalter, also ein medizinischer Laie, auch für Maßnahmen wie das Anlegen der Zwangsjacke oder die Isolierung unruhiger Kranker zuständig gewesen. Das völlige ärztliche Primat hat sich freilich nicht überall durchgesetzt, und daß dies schlimme Folgen haben konnte, zeigte sich besonders $\mathrm{kra}$ am sogenannten «Burghölzliskandal» von 1878/79. Dieser hatte nur das Gute, daß damit der noch sehr junge August Forel Direktor wurde, der sich zudem nur aus rein persönlichen Gründen damals nicht scheute, sich in ein Wespennest zu setzen ${ }^{20}$.

c) Die Behandlung der Geisteskranken. Der Doppelsinn des Ausdruckes «Behandlung» hat für die Psychiatriegeschichte eine besondere Bedeutung: Die Erfüllung humanitärer Pflichten hat schon bei Pinels Reform auch zu einer besseren Therapie geführt, und das wiederholte sich ein halbes Jahrhundert später beim No-restraint. Ludwig Wille schrieb, daß dieses zu einer intensiveren Beschäftigung mit dem Kranken zwinge; HeINRICH Cramer erzielte bei der Verbannung aller Zwangsmittel aus seiner Anstalt Erfolge, die noch heute imponieren. Die - an sich freilich nicht neue ${ }^{21}-$ Arbeitstherapie wurde konsequent gehandhabt, und Wille berichtete nicht ohne Stolz, daß rund $70 \%$ seiner Patienten beschäftigt würden - und das in einer Pflegeanstalt. Der freie Ausgang wurde möglichst vielen Kranken gewährt, ebenso Urlaube von wochenlanger Dauer. Angehörige wurden zu Besuchen ermuntert. Was heute in einer guten Klinik selbstverständlich ist, war es damals jedenfalls in den fortschrittlichsten auch schon. Die neuen Behandlungsformen ließen erkennen, daß gewisse psychische Endzustände nicht Folge der Krankheit, sondern Folge schlechter Behandlung waren, so daß sich bei Wille bereits eine Erwähnung des psychischen Hospitalismus findet ${ }^{22}$.

d) Zur fürsorgerischen Betreuung entlassener Patienten wurde wenigstens durch Gründung von Hilfsvereinen für Geisteskranke ein Anfang gemacht. Solche wurden z.B. von August Zinn und von Eduard Hitzig gegründet.

19 A.ZInN, Die öffentliche Irrenpflege im Kanton Zürich (etc.), Zürich.

20 A. FoneL, Briefe/Correspondance 1864-1927, Bern/Stuttgart 1968.

21 R. Herzog und H. WALSER, Johann Konrad Fäsi-Geßner (1796-1870), Zürcher Taschenbuch auf das Jahr 1969 (Fäsi war Verwalter des alten Spitales, einschließlich des alten Irrenhauses).

22 Hans H. WaLSER, Hundert Jahre Klinik Rheinau 1867-1967, Verlag Sauerländer, Aarau 1970. 
e) Schulung des Pflegepersonales wurde gefordert, dieses Postulat wurde aber erst nach 1900 allmählich erfüllt. Etwas besser stand es mit dem psychiatrischen Unterricht für Studenten: Hier erreichte es August Forel immerhin im Jahre 1888, daß die Psychiatrie Prüfungsfach wurde.

\section{Griesingers neue Vorschläge von 1868}

Wilhelm Griesingers beweglicher, suchender Geist stellte das kaum Erreichte schon 1868 wieder in Frage ${ }^{23}$. Er wenigstens merkte, daß mit dem neuen Anstaltstypus eine ganze, recht umfassende Konzeption des «Irrenwesens » gleichsam unwillkürlich entstanden war, und er konnte ihrer nicht froh werden. Sein erster Vorschlag war, an die Stelle des (ohnehin fraglichen) Kriteriums der Heilbarkeit oder Unheilbarkeit dasjenige der Dauer des Klinikaufenthaltes zu setzen. Das mußte, nach seinen Worten, zu total verschiedenen Einrichtungen führen: «... weit verschiedener, als bisher die Heil- und Pflegeanstalten unter sich differieren; nur für die eine, nämlich für die ... Bestimmung eines langen Aufenthaltes sind diejenigen speciellen Institute erforderlich, die man heutzutage allgemein meint, wenn man von modernen Irrenanstalten spricht». Für transitorische Aufenthalte sollte jede Stadt eine Klinik haben, wo Patienten rasch, ohne große Formalitäten, gratis oder zu kleinem Verpflegungssatz aufgenommen werden könnten. Diese Klinik sollte klein, aber mit reichlich wissenschaftlich gut ausgebildetem Personal dotiert sein; womöglich sollte eine besondere ärztliche Indikation für die Aufnahme gerade in diese Klinik gestellt werden. Diese sollte auch dem Unterricht dienen, und möglichst nahe bei einem allgemeinen Krankenhaus liegen. Vorteile dieses «Stadt-Asyles» wäre die Nähe der Familie, die Möglichkeit, die Patienten tagsüber in der Stadt arbeiten zu lassen, und damit die Begünstigung einer raschen Rückkehr ins normale Leben. Bei längerer Krankheitsdauer sollten die Patienten in «ländliche Asyle» eintreten, die ungefähr den bestehenden Anstalten entsprechen würden. Ein besonderes Anliegen Griesingers war eine dritte Behandlungsform, die «freie Verpflegung». Sie sollte stets mit einer Anstalt kombiniert, also ärztlich geleitet sein. Er schlug zwei Formen vor: Zunächst die «agricole Colonie», wobei die Kranken in Feld und Garten arbeiten würden, und dann die «familiale Verpflegung». Dabei würden die Kranken als Pensionäre z.B. in Handwerkerfamilien aufgenommen, zudem sollten neue Siedlungen -

23 W. GRIESINGER, Über Irrenanstalten und deren Weiter-Entwicklung in Deutschland, in Gesammelte Abhandlungen, 1. Band, Berlin 1872. 
analog den damals neuen Arbeitersiedelungen - errichtet werden. Eine Selbstverwaltung durch die Kranken sah er allerdings noch nicht vor, die Siedelungen sollten vielmehr von geschulten Pflegern geleitet werden. Diese Behandlungsformen, so schrieb Griesinger, kämen nicht teurer, dem Kranken würde «mit dem Billigeren auch das Bessere» geboten, außerdem das, «was die prachtvollste und bestgeleitete Anstalt der Welt niemals gewähren kann, die volle Existenz unter Gesunden, die Rückkehr aus einem künstlichen und monotonen in ein natürliches sociales Medium, die Wohltat des Familienlebens ». - Man muß freilich eine Ahnung vom eintönigen Leben eines Patienten in einer Anstalt alten Stiles haben, um das Revolutionäre an Griesingers Vorschlägen ganz würdigen zu können.

Griesinger schlug also nichts weniger als eine neue Gesamtkonzeption vor, durch welche der Geisteskranke von Anfang an wieder in die Gesellschaft reintegriert oder womöglich gar nicht erst aus seiner sozialen Umgebung gelöst würde. Die Anstalten würden zwar bestehen bleiben, jedoch nur als eine der Behandlungsformen, und nicht einmal als die wichtigste. Der Anstaltsdirektor wäre um seine absolute Macht gebracht worden. Der neue Anstaltstypus, den man nun erst mühevoll geschaffen hatte und der für Jahrzehnte eine neue Grundlage zu geben versprach, sollte nun schon wieder revidiert werden. Die Aufnahme von Griesingers Vorschlägen war deshalb geteilt. Wortführer der konservativen Gruppe war H.LAEHR in Berlin, und seine Sprache war deutlich, nicht weniger heftig aber war die Replik Griesingers ${ }^{24,25}$. Bei den jungen, aufgeschlossenen Psychiatern aber fand Griesinger Zustimmung. Seine Gedanken haben wie ein Ferment gewirkt, bis hin zu Eugen Bleuler. Verwirklicht wurde aber wenig, das Bleigewicht der etablierten Institution ließ einen weiteren, entscheidenden Fortschritt nicht zu. Zögernd wurde allmählich dies und jenes in die Tat umgesetzt, so im Kanton Zürich 1908 die Familienpflege. Die Stagnation in der praktischen Psychiatrie mag dazu beigetragen haben, daß sich August Forel mehr psychohygienischen und prophylaktischen Fragen zuwandte und daß sich Eugen Bleuler besonders intensiv mit Psychpathologie beschäftigte.

24 H.Laehr, Fortschritt? - Rückschritt! Reform-Ideen des Herrn Geh. Rates Prof. Dr. Griesinger in Berlin auf dem Gebiete der Irrenheilkunde, Berlin 1868.

25 W. Griesinger, Zur Kenntnis der heutigen Psychiatrie in Deutschland, Eine Streitschrift, Leipzig 1868. Die letzten Sätze der Broschüre sind: «Es war Ehrensache für mich, diese Angriffe abzuwehren und nothwendig, über das Treiben des Herrn Laehr das ärztliche Publikum aufzuklären ... Man kennt ihn jetzt - den Samariter mit der Zwangsjacke.» 


\section{Zusammenfassung}

Die «Schweizer psychiatrische Schule», die von August Forel und von Eugen Bleuler etwa zwischen 1880 und 1920 begründet wurde, hat eine bisher wenig beachtete, aktive und ideenreiche Periode als geschichtlichen Vorläufer. Damals wurde der Anstaltstypus geschaffen, der bis heute die Grundlage der praktischen Psychiatrie geblieben ist. Die Aufbauarbeit wurde besonders von deutschen, in unserem Lande tätigen Psychiatern geleistet, weshalb für die Zeitspanne vom Beginn der Anstaltsgründungen bis zu August Forel (etwa 1850-1880) die Bezeichnung «Deutsche Periode der Schweizer Psychiatrie» vorgeschlagen wird. Bemerkenswert ist, daß Wilhelm Griesinger, der bedeutendste dieser Pioniere, schon recht früh (1868) das Erreichte durch eine neue Gesamtkonzeption wieder in Frage zu stellen versuchte. Er wollte den Kranken möglichst weitgehend in seiner sozialen Umgebung belassen oder ihn doch rasch wieder in die Gesellschaft integrieren. Davon wurde damals wenig verwirklicht, doch scheinen seine Vorschläge nun im erweiterten Rahmen der modernen Sozialpsychiatrie endlich ihre Verwirklichung zu finden. 